\title{
Different Histogenesis of Experimental Odontogenic Cysts by the Renal Subcapsular Transplantation of Tooth Germs of Mice
}

\author{
En-Xin Zhu' ${ }^{1}$ J ing Xiao', Ting-J iao Liu' ${ }^{1}$, Wei-Wen J iang ${ }^{2}$ and Minoru Takagi ${ }^{2}$ \\ ${ }^{1}$ Department of Oral Pathol ogy, Faculty of Dentistry, Dalian Medical University, Dalian, Republic of China, ${ }^{2}$ Mol ecular Pathol ogy, De- \\ partment of Oral Restitution, Division of Oral Health Sciences, GraduateSchool, Tokyo Medical and Dental University, Tokyo, J apan
}

\begin{abstract}
Zhu EX, Xiao J , Liu TJ , J iang WW and Takagi M. Different Histogeneses of Experimental Odontogenic Cysts by the Renal Subcapsular Transplantation of Tooth Germs of Mice. Oral Med Pathol 2000; 5: 83-86, ISSN 1342-0984.
\end{abstract}

\begin{abstract}
The tooth germs of first mandibular molars of 17.5-day-old C3H mouse embryos were transplanted into the renal subcapsular spaces of 3-month-old syngenic male mice. Grafts were harvested at timed sequences from 1, 2, 3, 4 weeks and 2, 3, 4, 5 months, and were examined histopathologically by total serial sections. Of 32 cases of grafts, keratocyst-like lesions were formed in 13 cases, and dentigerous cyst-like lesions were formed in 7 cases. None of the cases developed two cysts at the same time. In the keratocyst-like lesions the cavity developed from the enamel epithelium in the early stage after transplantation, and in the dentigerous cyst-like lesion the cavity developed by cystic degeneration of stellate reticular layer of enamel organ in the late stage after transplantation. The present experiment revealed that keratocyst-like lesions and dentigerous cyst-like lesions developed through different histogeneses.
\end{abstract}

Key words: animal model, odontogenic cysts, tooth germ, transplantation

Correspondence: Wei-Wen J iang, Molecular Pathology, Department of Oral Restitution, Division of Oral Health Sciences, Graduate School, Tokyo Medical and Dental University, 1-5-45, Yushima, Bunkyo-ku, Tokyo, 113-8549, J apan

Tel: +81-3-5803-5453 Fax: +81-3-5803-0188 E-mail: ww.mpa@tmd.ac.jp

\section{Introduction}

Odontogenic cysts in human beings are not uncommon. There are many types displaying various biologic behaviors, but little is known about their histopathogenesis. It is generally considered that each of the odontogenic cysts is derived from one of the following epithelial sources: 1) enamel organ, 2) reduced enamel epithelium of a tooth crown, 3) epithelial rests of Malassez and remnants of the sheath of Hertwig, 4) remnants of the dental lamina, or 5) basal cells of oral mucosa $(1,2)$. However, the exact relationship between these epithelia and various types of cysts is unknown, and experimental studies in this field have been limited. In order to investigate whether enamel organ is one of the sources of odontogenic cysts, and which type of odontogenic cyst is closely connected with it, we performed the following animal experiment.

\section{Materials and Methods}

$\mathrm{C} 3 \mathrm{H} / \mathrm{HeN}$ mice were used. They were given mixing solid food and sterile drinking water. Two-month-old mice of both sexes mated at 9 o'd ock at night and before 9 o'clock the next morning. The mice which had vaginal plugs were recognized as becoming pregnant after 0.5 day.
And on day 17.5, the tooth germs of the embryos were in the bell stage, in which a specialization of cells of the enamel organ itself occur, resulting in the establishment of four distinct epithelial layers: external enamel epithelium, stel late reticulum, stratum intermedium, and internal enamel epithelium. The 17.5-day embryos were re moved from pregnant females and placed in $2 \%$ bovine serum in Hanks solution. Under a dissecting microscope,

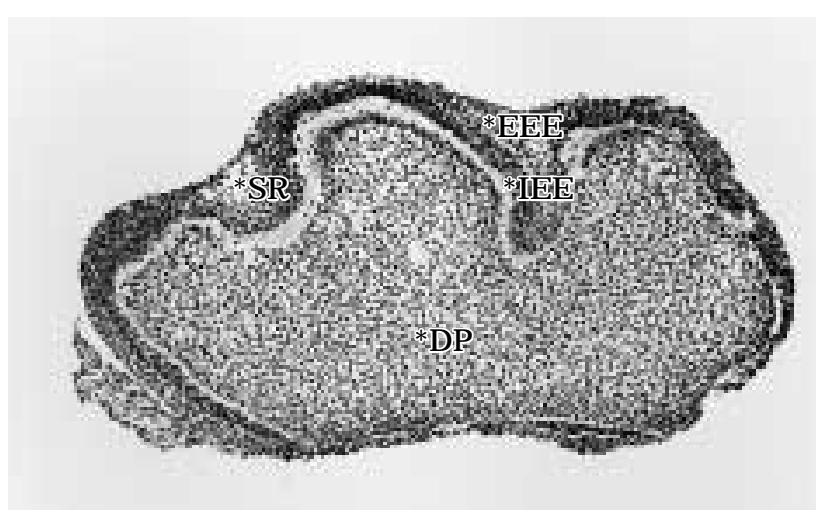

Fig. 1: First mandibular tooth germ as removed from a 17.5-dayold mouse. IEE: internal enamel epithelium, EEE: external enamel epithelium, DP: dental papilla SR: stellate reticulum. H.E. $\times 20$ 
the first mandibular molar tooth germs of these embryos were dissected from the surrounding connective tissues, dental Iamina and oral epithelium. The tooth germs which were just in the bell stage, had only components of enamel organ, dental papilla and dental follicle, and not other tissues, such as dental lamina and oral epithelium (Fig. 1). Three-month-old syngenic male mice were anaesthetized by the intraperitioneal injection of pentobarbital sodium (Sommonpentyl), and 32 dental germs were transplanted under the left renal subcapsular spaces of the mice at the rate of one graft per capita. The grafts were harvested 1, 2, 3, 4 weeks and 2, 3, 4, 5 months after transplantation. For histological examination, the grafts were fixed in $10 \%$ neutral buffered formalin and demineralized in $5 \%$ formic acid at $4^{\circ} \mathrm{C}$ for 12 hours. Routinely the paraffin-embedded specimens were serially sectioned 4 $\mu \mathrm{m}$ thick and stained with hematoxylin-eosin.

For determining the relationship between duration of transplantation and formation of experimental odontogenic cysts, pearson chi-squared test ( $\chi^{2}$ test) was applied.

\section{Results}

1) Histopathol ogical Findings

(1) One-week group

At 1 week after transplantation, small amounts of dentin and enamel appeared in the bell stage, tooth germs and stellate cells of enamel organs were still abundant. External enamel epithelium proliferated partially and the serial sections showed that these proliferations were sticking out of the enamel organs and were still connected with enamel organs by epithelial strands (Fig. 2). Formation of the inner space with hyperkeratinization was observed in these epithelial nest (Fig. 3)

(2) Two-week group

At 2 weeks after transplantation, the enamel and dentin of the tooth crown were almost formed and the stellate reticulum layer disappeared. The size of epithe lial nests enlarged and keratocyst-like lesions were filled with keratin. The inner surface of cysts was lined by with keratinized or parakeratinized stratified squamous epithelium. Basal layer of stratified squamous epithelium was smooth without rete pegs (Fig. 4).

(3) Thre-wek group

At 3 weeks after transplantation, the crown was formed and the root began to develop. Keratocyst-like le sions continuously increased in size and daughter-cysts were sometimes seen. Alveolar bone developed and bone marrow appeared.

(4) Four-week group

At 4 weeks after transplantation, parts of enamel organs enlarged and bulged outwards and a cystic cavity was formed. There were dentigeous cyst-like lesions, and the epithelium of the cystic lining was composed of 2 to 4 layers of non-keratinized stratified squamous epithelium. Basal layer of the epithelium was flat and rete pegs were not formed (Fig. 5). The cystic cavity was filled with a fluid, desquamated epithelial cells and foamy cells.

\section{(5) Two-to-threemonth group}

Dentigerous cyst-like lesions were lined with 2 to 4 layers of flattened non-keratinized stratified squamous epithelium. The crown of the tooth was enclosed in a cys-

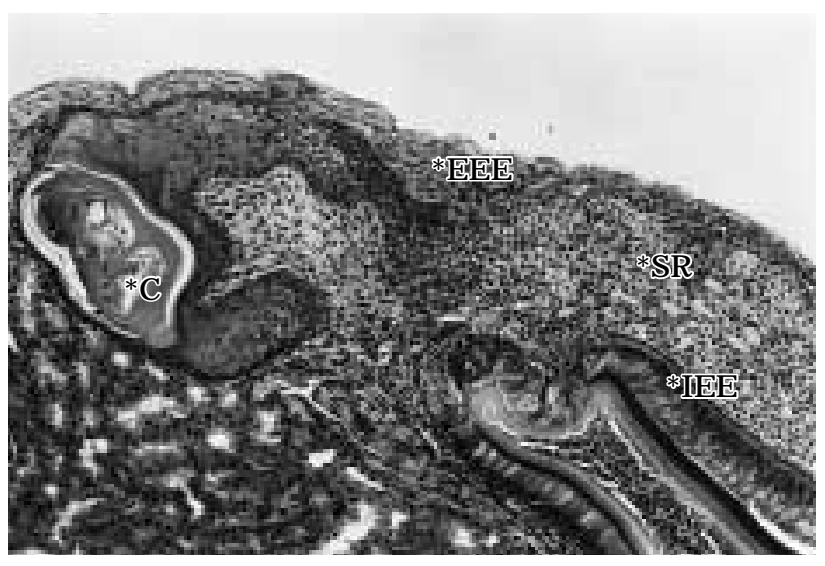

Fig. 2: one-week transplantation. The cyst was connected with enamel organ by epithelial strand. IEE: internal enamel epithelium, EEE: external enamel epithelium SR: stellate reticulum, C: cyst. H.E. $\times 10$

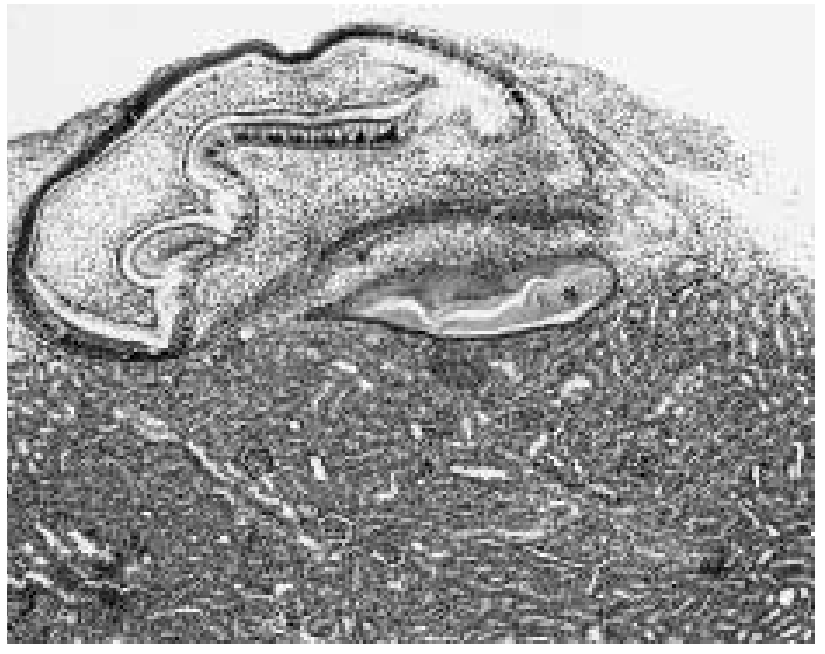

Fig. 3: One-week transplantation. Formation of inner space with hyperkeratinization is observed in the epithelial nest $\left(^{*}\right)$. H.E. $\times 80$

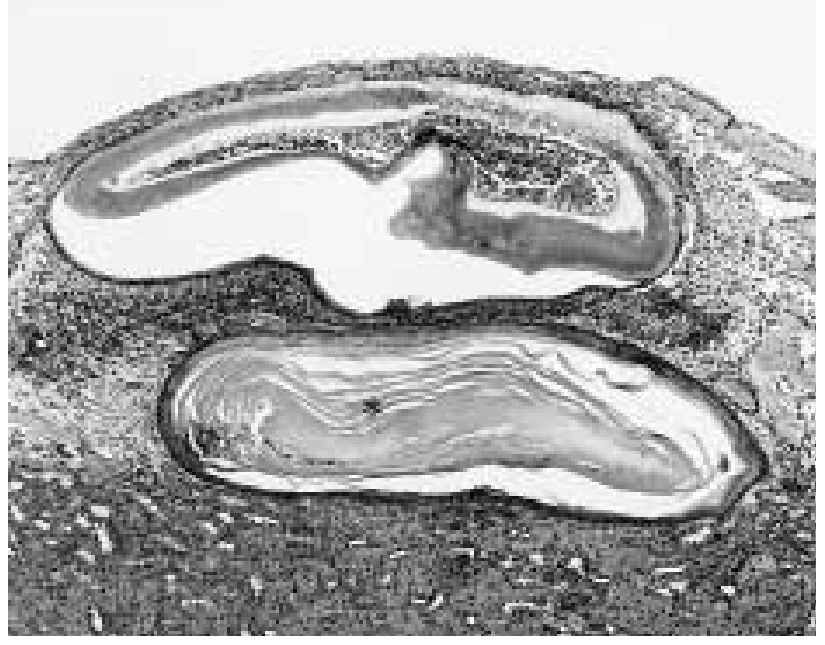

Fig. 4: Two-week transplantation. Keratocyst-like lesion is formed outside of tooth germ and filled with keratin $(*)$. H.E. $\times$ 160 
tic cavity and the cavity was filled with a fluid.

(6) Four-to-fivemonth group

The dentigerous cyst-like lesions continued to develop in size (Fig. 6). The lining epithelium was thin nonkeratinized stratified squamous epithelium without rete pegs and was attached to the cementoenamel junction of the unerupted tooth (Fig.7) In some tooth germs, large amounts of cementum-like hard tissue was formed. The cystic cavity was filled with a faintly eosinophilic fluid and scanty cells. In the reduced enamel epithelium, a cuticle-like eosinophilic substance and irregular calcification were seen. Large amounts of alveolar bone formation and bone marrow tissue were seen.

2) Relationship between duration of transplantation and formation of odontogenic cyst

The findings in experimental transplants are summarized in Table 1. Thirteen cases of 32 transplants devel oped keratocyst-like lesions during 1 to 4 weeks, and 7 cases developed dentigerous cyst-like lesions during 2 to 5 months. There was also a tendency for keratocyst-like lesions to develop in the early stage and the dentigerous cyst-like lesions to develop in the late stage $(\mathrm{P} \varangle 0.05$ ).

Table 1: Relationship between duration of transplantation and formation of odontogenic cysts

\begin{tabular}{ccccc}
\hline $\begin{array}{c}\text { Duration of } \\
\text { transplantation }\end{array}$ & $\begin{array}{c}\text { Transplanted } \\
\text { cases }\end{array}$ & $\begin{array}{l}\text { Keratocyst-like } \\
\text { lesions }\end{array}$ & $\begin{array}{l}\text { Dentigerous } \\
\text { cyst-likelesions }\end{array}$ & $\begin{array}{c}\text { Total } \\
\text { 1-2 weeks }\end{array}$ \\
\hline 3-4 weeks & 8 & 8 & 0 & 8 \\
2-3 months & 8 & 1 & 2 & 3 \\
4-5 months & 8 & 0 & 2 & 2 \\
\hline Total & 32 & 4 & 3 & 7 \\
\hline
\end{tabular}

\section{Discussion}

It is well known that tooth germs continue to devel op even in transplanted conditions (3-6). Thus it is reasonable to study histogenesis of odontogenic cysts by using transplanted tooth germs. In human beings, each of the odontogenic cysts have different histological features and biological behaviors, and it can be supposed that they have different epithelial origins. According to the development of keratocysts, two sources of the epithelium have been considered; remnants of the dental lamina and extensions of basal cells from the overlying oral epithelium $(7,8)$. In animal experiments, it has been demonstrated that the cervical loop and dental lamina were the sources of odontogenic keratocyst (9). Experimental odontogenic keratocysts by transplantation have been reported by Bartlett et al . and Soskolne et al . (10, 11). The histology of the cysts in these experiment was the similar to those seen in ours. In the present experiment, the serial sections from 13 cases of keratocyst-like lesions showed that the epithelial nests proliferated and finally keratocystlike lesions were formed. F ormation of keratocyst-like le sion was not seen within enamel organs. In those lesions, epithelial lining of the cysts were connected with external enamel epithelium which developed toward surrounding connective tissues. Therefore, it is suggested that exter-

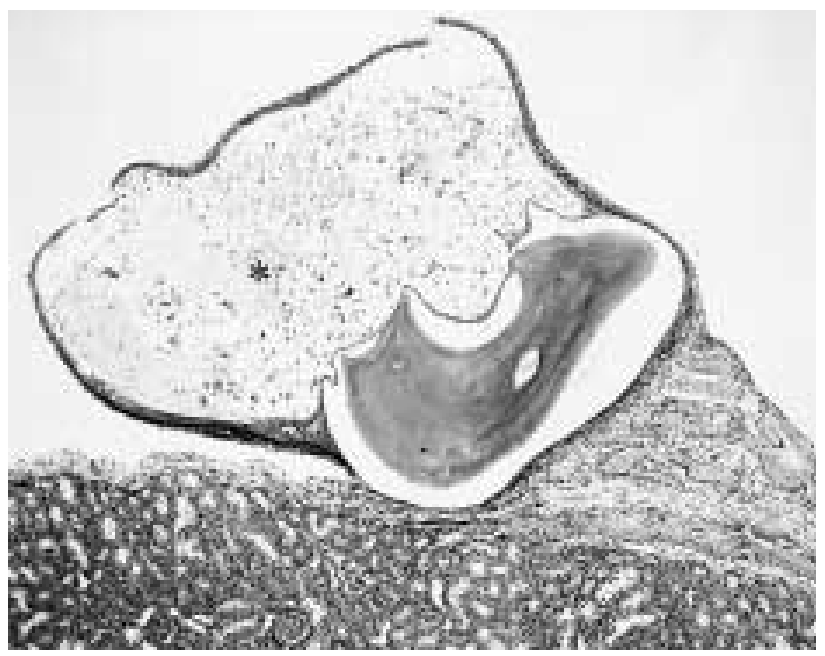

Fig. 5: Four-week transplantation. Dentigerous cyst-like lesion is lined with non-keratinized stratified squamous epithelium $(*)$. The cystic cavity is filled with liquid, desquamated epithelial cells and infiltrating cells. H.E. $\times 72$

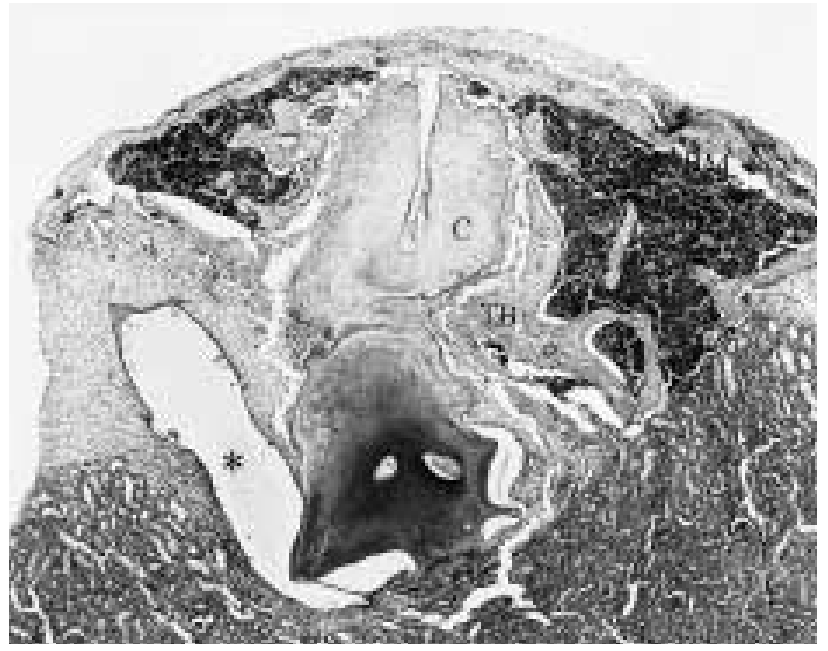

Fig. 6: Five-month transplantation. Dentigerous cyst is attached to amelocemental junction of the tooth $(*)$. Large amount of cementum-like (C) hard tissue is formed. TB: trabecular bone, BM: bone marrow H.E. ×50

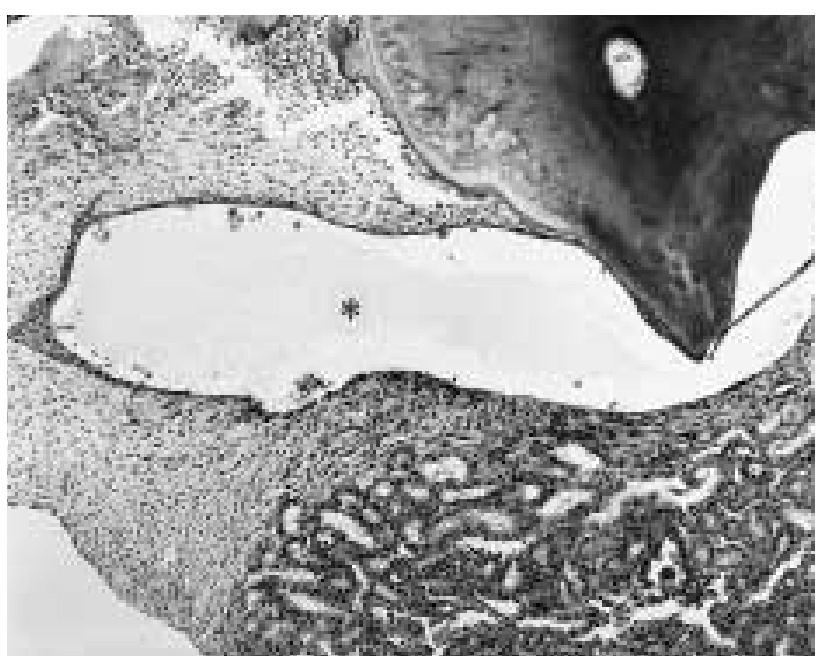

Fig. 7: Five-month transplantation. Higher magnification of Fig. 6. Cyst is lined by non-keratinizing stratified squamous epithelium. H.E. $\times 160$ 
nal enamel epithelium is the sources of keratocyst-like lesions. However the biological behaviors of the keratocystlike lesions were considered to be different from odontogenic keratocysts of humans. The keratocyst-like lesion in the present experiment did not show aggressive growth.

Dentigerous cysts may develop by accumulation of fluid either between the reduced enamel epithelium and the enamel or within the enamel organ itself. Atkinson described the formation of cysts derived from the enamel organ around the crowns of mouse molar teeth transplanted subcutaneously into an inbred strain of mice (12). $\mathrm{He}$ also examined proliferative populations of cells by ${ }^{3} \mathrm{H}$ labelling method in the experimental odontogenic cysts (13). In the present experiment, the dentigerous cyst-like lesions occurred in the stellate reticular layer. At first cellular degeneration and necrosis appeared within the stellate reticular layer, and a fluid accumulated. The cysts were attached to cementoenamel junctions, showing a tooth-containing or half-tooth-containing appearance. In those cases proliferation of external enamel epithelium was not observed. It is suggested that the enamel organ is one of the sources of dentigerous cyst-like lesions. Experimental dentigerous cysts have been reported by Riviere et al ., Al-talabani et al . and Alini et al . (14-16), and they suggested the same histogenesis.

In the present experiment, most of keratocyst-like lesions were observed during 1 to 4 weeks, and dentigerous cyst-like lesions developed during 2 to 5 months. None of the cases developed the two cysts at the same time. It is supposed that keratocyst-like lesions were formed in the early stage of tooth development, and dentigerous cyst-like lesion were formed in the late stage $(\mathrm{P}<$ 0.05).

Atkinson described that the initial loss of blood supply and subsequent establishment of vascular connection between host and graft influenced the pattern and types of degeneration and repair (12). Formation of experimental odontogenic cysts by transplantation without any specific pretreatment has been reported by several authors (11-16). Maeda et al . reported that mechanical injury was an important factor in the production of odontogenic carcinoma by $\mathrm{N}$-ethyl-N-nitrourea (17). However Miura et al. considered that in vitro 4-nitroquinoline-1-oxide (4 NQO) treatment is necessary for induction of experimental odontogenic cysts (18). In the present experiment, the injury factors might have three sources. The first is the procedure of removing the tooth germ from pregnant females and dissecting it from the surrounding tissues. The second might come from the pressure of the renal capsule. The third factor may be connected with the orientation of the tooth germ under the renal capsule since the orientation has something to do with the resistance of the surrounding tissues to the tooth germ.

\section{Acknowledgment}

This work was supported by a Grand-in Aid from the) apan China Medical Association.

\section{References}

1. Shafer WG, Hine MK and Levy BM. A Textbook of Oral Pathology. 4 th ed. W.B. Saunders. Philadel phia. 1983; 258-75.

2. Shear M and Seward GR. Cysts of the Oral Regions. 3rd ed. Oxford, London. 1992; 85.

3. Hay MF. The development in vivo and in vitro of the lower incisor and molars of the mouse. Arch Oral Biol 1961; 3: 86109.

4. Atkinson ME. A histological study of tooth grafts in an inbred strain of mice. J Oral Pathol 1972; 1: 115-24.

5. Al-Talabani NG and Smith CJ . Continued development of 5day old tooth-germs transplanted to syngeneic hamster (mesocricetus auratus) cheek pouch. Arch Oral Biol 1978; 23: 1069-76.

6. Morio I. Recombinant study of the mouse molar cervical loop and dental papilla by renal transplantation. Arch Oral Biol 1985; 30: 557-61.

7. Harris $\mathrm{M}$ and Toller $\mathrm{P}$. The pathogenesis of dental cysts. $\mathrm{Br}$ Med Bull 1975; 31: 159-63.

8. Brannon RB. The odontogenic keratocyst: A clinicopathologic study of 312 cases. Part II. Histologic features. Oral Surg Oral Med Oral Pathol 1977; 43: 235-55

9. Zhu E-X. Dental lamina as presumptive source of odontogenic cyst. J Stomatol Soc J pn 1990; 57: 549-61. (In J apanese with English abstract)

10. Bartlett PF, Radden BG and Reade PC. The experimental production of odontogenic keratocysts. J Oral Pathol 1973: 2: 58-67.

11. Soskolne WA, BabJ and Sochat S. Production of keratinizing cysts within mandibles of rats with autogenous gingival epithelial grafts: A histologic study. J Oral Pathol 1976: 5: 122-8.

12. Atkinson ME. A histological study of odontogenic cysts formed following mouse molar tooth transplantation. J Oral Pathol 1976; 5: 347-57.

13. Atkinson ME. An autoradiographic study of experimental odontogenic cyst formation in the mouse. J Oral Pathol 1977; 6: 382-6.

14. Riviere GR and Sabet TY. Experimental follicular cysts in mice: A histologic study. Oral Surg Oral Med Oral Pathol 1973; 36: 205-13.

15. Al-Talabani NG and Smith CJ. Experimental dentigerous cysts and enamel hypoplasia: their possible significance in explaining the pathogenesis of human dentigerous cysts. J Oral Pathol 1980; 9: 82-91.

16. Altini $M$ and Cohen $M$. Experimental extra-follicular histogenesis of follicular cysts. J Oral Pathol 1987; 16: 49-52.

17. Maeda H, Kameyama Y, Fujita K, et al . Experimental odontogenic tumors produced by ethylnitrosourea injections and mechanical injuries. J Oral Pathol Med 1991; 20: 296-9

18. Miura Y, Ozaki HS, Li T-J , et al . Experimental odontogenic cysts induced by in vitro 4-nitroquinoline 1-oxide (4 NQO) treatment of F 344 rat incisor tooth germs. J Oral Pathol Med 1998; 27: 53-8.

(Accepted for publication October 16, 2000) 